\title{
Marco teórico del efecto de interferencia en contextos neutrales y emocionales
}

\section{Interference Effect in neutral and emotional contexts: a theoretical framework}

\author{
Eliana Vanesa Zamora, Isabel Introzzi, Macarena del Valle y María Richard's \\ Instituto de Psicología Básica, Aplicada y Tecnología (IPSIBAT), Consejo Nacional de Investigaciones Científicas \\ y Técnicas (CONICET) y Universidad Nacional de Mar del Plata (UNMDP), Argentina.
}

\begin{abstract}
Resumen
La interferencia es el efecto experimental causado por información distractora que se manifiesta en una disminución del desempeño respecto a condiciones que implican ausencia de información distractora. Si bien existe un notorio cuerpo de literatura respecto a este efecto, la gran mayoría de los trabajos existentes lo analizan en contextos neutrales, es decir, sin saliencia emocional. Por este motivo, en este trabajo se revisará el efecto de interferencia, sus dimensiones temporales y formales, como así también los contextos en los que interviene, es decir no sólo los neutrales sino también aquellos con saliencia emocional. Con respecto a estos últimos, se revisan los diferentes aspectos de los contextos emocionales que modulan el efecto de interferencia. Finalmente, se incorporan diferentes nociones que han inclinado la balanza a favor del efecto de interferencia como el principal mecanismo explicativo de los procesos de control inhibitorio.
\end{abstract}

\begin{abstract}
Interference is the experimental effect caused by distracting information. It manifests as decreased performance in conditions with distracting information compared to performance in conditions without distracting information. Although many studies have investigated this effect, most of them have analysed it in neutral settings (i.e. settings without emotional salience). We review the interference effect, its temporal and formal dimensions, and settings in which it occurs (i.e. in neutral as well as emotionally charged settings), and different aspects of emotional settings that modulate the interference effect. Finally, we include different concepts that support the interference effect as the main explanatory mechanism of inhibitory control processes.
\end{abstract}

Key Words: interference; inhibition; emotion; emotional stimuli.

Palabras Clave: interferencia; inhibición; emoción; estímulos emocionales.

Cómo citar este artículo: Zamora, E., Introzzi, l., del Valle, M., \& Richard's, M. (2020). Marco teórico del efecto de interferencia en contextos neutrales y emocionales. Escritos de Psicología - Psychological Writings, 13(1), 23-33. https://doi.org/10.24310/espsiescpsi.v13i1.10079

Correspondencia: Eliana Vanesa Zamora. Instituto de Psicología Básica, Aplicada y Tecnología (IPSIBAT), Consejo Nacional de Investigaciones Científicas y Técnicas (CONICET) y Universidad Nacional de Mar del Plata (UNMDP), Argentina. E-mail: elianavanesazamora@gmail.com. E-mail de la coautora Isabel Introzzi: isabelintrozzi@gmail.com. E-mail de la coautora Macarena del Valle: mdelvalle1989@gmail.com. E-mail de la coautora María Richard’s: mariamartarichards@gmail.com. 


\section{Introducción}

El término interferencia se refiere a un efecto experimental clásico causado por información distractora que se manifiesta a través del incremento en el Tiempo de Respuesta (TR) respecto a una línea de base previa que implica la ausencia de información distractora. Si bien existe un notorio cuerpo de literatura respecto de este efecto e incluso se plantea en los orígenes de la psicología cognitiva, los estudios que analizan el efecto experimental de interferencia en contextos emocionales son limitados. Incluso las revisiones sobre la interferencia realizan énfasis en los aportes de las neurociencias (Compton, 2003), de la neuropsicología (Song et al., 2017), y en distintos trastornos psicopatológicos como la ansiedad (Boehme et al., 2015), trastornos del aprendizaje (Gu et al., 2019), y en muestra clínica en general (Broome et al., 2015), sin analizar de manera exhaustiva el efecto experimental de interferencia y sus modalidades. Por ello este trabajo pretende mediante una revisión no sistemática -también denominada bibliográfica- reunir y analizar: (1) los principales aportes del efecto experimental de la interferencia, y (2) los factores a considerar para generar el efecto de interferencia en tareas experimentales.

\section{Acerca del efecto de interferencia}

Según McLeod (2007) el término interferencia debe reservarse para explicar el fenómeno empírico por el que disminuye el rendimiento, y considerar que en sí misma la interferencia no requiere supresión de contenidos ni de procesos cognitivos. En otras palabras, la interferencia también puede definirse como la competencia cognitiva entre estímulos, procesos o respuestas que dan lugar a un deterioro en la ejecución de una actividad (Harnishfeger, 1995). De este modo, la interferencia afecta a la rapidez con que se realiza el procesamiento cognitivo, ya que produce un embotellamiento de información durante el cual se debe decidir qué información será procesada y qué respuesta será ejecutada. La interferencia y la resistencia a la interferencia son procesos multifacéticos, indicando que la funcionalidad de la interferencia varía a lo largo de dos dimensiones principales: la dimensión temporal y la dimensión formal (Dempster, 1993).

Desde su dimensión temporal, la interferencia puede obrar en tres sentidos: como Interferencia Proactiva (IP), como Interferencia Retroactiva (IR) o como Interferencia Coactiva (o concurrente, IC). La IP se observa cuando la información aprendida previamente interfiere con la información aprendida más tarde. En contextos de laboratorio se ha encontrado que la IP varía en función de la cantidad de información presentada previamente, y además por su similitud gramatical, visual o semántica (Underwood, 1957). Se ha señalado que la IP puede ocurrir principalmente por dos motivos: debido a problemas en la recuperación de la respuesta correcta (discriminabilidad), o por la fuerza de activación de la respuesta incorrecta aprendida inicialmente (Jacoby, Debner y Hay, 2001). Por lo tanto, la IP puede producirse porque la respuesta correcta es muy débil o porque la respuesta incorrecta es muy fuerte. Por otro lado, la IR se genera cuando la información nueva provoca dificultades para recordar información previamente aprendida (Klein, 1964). En otras palabras, el conocimiento posterior podría contribuir al olvido y ocurriría principalmente cuando la información previa no se ha puesto en práctica o recordado durante algún periodo variable de tiempo (Edwards, 2010). Por ejemplo, resulta habitual olvidar la contraseña de un correo electrónico en desuso porque producen interferencia las contraseñas más recientes y más utilizadas. Cabe destacar la línea de trabajo de Mark Bouton (1993, 2018), que señala que la IR no contribuye directamente al olvido, sino que la información presentada previamente se hace contexto-dependiente. Según el autor, cuando los participantes experimentan IR, su atención empieza a trasladarse, no sólo a los propios estímulos, sino también al contexto en el que estos estímulos aparecen, dificultando la recuperación. Asimismo, se ha encontrado que cuanto mayor sea la similitud entre el nuevo aprendizaje y el recuerdo de la información previamente adquirida, mayor es el efecto de la IR (McGeogh y McDonald, 1931).

Finalmente, la IC se produce cuando la información irrelevante se encuentra presente al momento de ejecutar la actividad principal, es decir que esta interferencia tiene lugar cuando dos o más eventos contiguos compiten entre sí; por ejemplo, encontrar un objeto específico entre un conjunto variado de objetos con características similares.

De las fuentes descritas de interferencia, la IP y la IR corresponden a contenidos que han sido previamente aprendidos o procesados mientras que, en la IC, la información que genera interferencia está presente al momento de ejecutar la actividad. De este modo podríamos discriminar entre la interferencia proveniente de información originada en un momento previo, e interferencia generada por información irrelevante presente.

Desde una perspectiva formal, la interferencia se clasifica en función de las etapas de procesamiento de la información: perceptiva, lingüística (o representacional) y motora (Dempster, 1993; van der 
Molen, 2000). En primer lugar, la interferencia perceptiva se refiere al efecto de interferencia generado por estímulos externos e irrelevantes para resolver la tarea que se está llevando a cabo y que pueden producir una disminución en el desempeño (Mishra, Anguera, Ziegler y Gazzaley, 2013). Una tarea clásica para medir este tipo de interferencia es la tarea de Flancos (Eriksen y Eriksen, 1974) donde el participante debe intentar ignorar los distractores y responder solamente al estímulo target.

Por otro lado, la interferencia lingüística, opera sobre las representaciones que han sido previamente activadas en la memoria de trabajo y que compiten con otras representaciones para la consecución de un objetivo (Casey, 2000). Si bien Dempster (1993) se refiere a este tipo de interferencia lingüística exclusivamente, las perspectivas sobre el formato de las representaciones mentales aluden a que las mismas pueden tener un formato lingüístico (o proposicional) o visuo-espacial. En este tipo de interferencia, las representaciones son irrelevantes para el objetivo actual, o incluso fueron relevantes en algún momento, pero debido al cambio de objetivos ya no lo son más. Esta interferencia, puede producirse en forma de pensamientos, ideas, significados ambiguos o inapropiados al contexto, irrelevantes de acuerdo al objetivo o tarea planteado.

Por último, la interferencia motora o entre respuestas motoras se refiere a la competencia entre respuestas que da lugar a un deterioro en la ejecución. Tradicionalmente, se presenta un conflicto entre una dimensión relevante y otra irrelevante: mientras que la dimensión relevante requiere una determinada respuesta, la dimensión irrelevante requiere de una respuesta incongruente o incluso opuesta a la requerida por la dimensión relevante. Por ejemplo, en tareas con efecto Simon (Simon y Berbaum, 1990), las respuestas del sujeto son más rápidas cuando el estímulo aparece del mismo lado del que debe emitirse la respuesta (respuesta ipsilateral al estímulo), que cuando lo hace del lado opuesto (respuesta contralateral al estímulo). La interferencia aparece cuando se debe dar una respuesta contralateral al estímulo, en donde el TR aumenta debido al conflicto entre dos respuestas motoras (Jones, Cho, Nystrom, Cohen, y Braver, 2002).

De este modo, tanto la interferencia motora como la perceptual son concurrentes debido a que la competencia entre estímulos y entre respuestas se da en el momento de ejecutar la respuesta o de prestar atención al estímulo target. Por otro lado, la interferencia lingüística, puede ser proactiva o retroactiva pero no concurrente, debido a que las representaciones deben haber ingresado al sistema cognitivo. Además de esta distinción clásica, actualmente suele considerarse que la interferencia puede provenir tanto del ambiente externo como interno (en forma de pensamientos intrusivos, emociones e impulsos), o como una interacción compleja entre estas dos fuentes, por ejemplo, cuando un estímulo externo (e.g., un estímulo amenazante) provoca una emoción (Ziegler, Janowich, y Gazzaley, 2018), incluso ha sido denominada interferencia emocional o producida en contextos emocionalmente salientes.

\section{Interferencia en contextos emocionales}

Los estímulos emocionales son aquellos estímulos capaces de desencadenar reacciones emocionales a un nivel fisiológico, subjetivo y/o comportamental (Brosch, Pourtois, y Sander, 2010). La mayoría de las teorías sobre emoción coinciden en señalar que un estímulo emocional representa un tipo especial de estímulo con alta relevancia para la supervivencia del sujeto, debido a que prepara al organismo de forma adaptativa y funcional para la acción. Entre estas teorías se distinguen dos enfoques principales: el enfoque discreto y el enfoque dimensional de las emociones (Mauss y Robinson, 2009). El primer enfoque propone que el procesamiento de los estímulos emocionales se organiza de forma categórica (e.g., miedo, ira, asco, tristeza, alegría y sorpresa), y que las respuestas presentan una forma prestablecida e innata, con un sentido evolutivo. Por otro lado, el enfoque dimensional enfatiza el rol de dos dimensiones bipolares: la valencia y el arousal. Mientras que la valencia indica la forma en que un individuo evalúa la bondad o cualidad de un estímulo (cuyo rango va desde lo agradable hasta lo desagradable, es decir de lo positivo a lo negativo), el arousal refleja la actividad que el estímulo genera sobre el sujeto que lo percibe (cuyo rango va desde la excitación a la calma). De esta manera, las emociones son consideradas fenómenos inespecíficos que se diferencian entre sí por la ubicación que tienen en cada una de estas dimensiones.

Las personas se encuentran continuamente expuestas a estímulos, que pueden ser de tipo emocional, producidos por eventos externos que incluso pueden modificar las sensaciones internas, sentimientos y respuestas de los sujetos. Cuando estos estímulos no son relevantes ni prioritarios para el desarrollo de la tarea en curso, es muy probable que interfieran con el funcionamiento cognitivo, conductual o afectivo (Iordan, Dolcos, y Dolcos, 2013). En general, la literatura se refiere al control de la interferencia emocional, como a la capacidad de controlar y minimizar el efecto perturbador de los estímulos emocionales cuando resultan irrelevantes (Cohen et al., 2016). Uno de los primeros desa- 
rrollos para analizar la interferencia producida por contenido emocionalmente saliente fue el Stroop emocional (Williams, Mathews, y MacLeod, 1996). En esta tarea los participantes deben nombrar lo más rápidamente posible el color de la palabra mientras ignoran su contenido (emocional). Mientras que la versión clásica del Stroop crea un conflicto entre un color incongruente con una palabra (e.g., la palabra rojo escrita en color azul), el Stroop emocional involucra palabras emocionalmente salientes y neutrales, cuyo color no genera conflictos con su significado (e.g, la palabra muerte en color azul). En este caso, el incremento de los TR se debe a que estas palabras captan la atención debido a su relevancia emocional (Yiend, 2010). Por ende, el tiempo que demora la persona en nombrar el color, se ha usado como una medida de cuántos recursos destina al procesamiento del contenido emocional de la palabra. Gotlib y McCann (1984) utilizaron por primera vez la tarea Stroop emocional en participantes clínicamente deprimidos, y encontraron que éstos presentaron mayores TR que los participantes del grupo control cuando debían nombrar el color de palabras de contenido emocional negativo que cuando las palabras eran de contenido positivo o neutral. Esto sugiere una mayor interferencia frente a las palabras emocionales negativas en esta población. Cabe destacar que más allá de sus diferencias, en ambas versiones de la tarea, se requiere la supresión de información irrelevante, ya sea emocionalmente saliente como en el Stroop emocional o neutral como en el caso del Stroop clásico (Compton et al., 2003).

Diversos estudios sugieren que el desempeño en diferentes tareas experimentales varía según se utilicen estímulos emocionales (e.g., caras amenazantes, imágenes desagradables) o neutrales (e.g., letras, números, formas geométricas) (ver revisión Yiend, 2010). Por ejemplo, como fue mencionado previamente en el caso del Stroop emocional, se observan mayores TR ante estímulos emocionales que ante estímulos neutrales, y estos TR han permitido cuantificar la cantidad de recursos cognitivos asignados y por lo tanto la interferencia que producen. Sin embargo, otros autores encontraron que este efecto puede observarse solamente en las diferencias de TR entre estímulos emocionalmente negativos y neutrales (Fox, Russo, Bowles, y Dutton, 2001; McKenna y Sharma, 2004). Pratto y John (1991) destacan que podría existir un procesamiento automático para detectar estímulos emocionales, y especialmente para aquellos estímulos que son negativos. Incluso actualmente, se ha señalado que el incremento significativo en los TR ante los estímulos negativos con respecto a los positivos en la tarea Stroop emocional se debería a que, los estímulos negativos, captan la atención de manera más rápida y por más tiempo, con lo que la tarea principal (identificar el color) se ve demorada (Song et al., 2017). A su vez, los estímulos negativos serían procesados preferencialmente por el valor adaptativo que implica atender y responder a este tipo de estímulos que pueden ser potencialmente peligrosos (Carretié, López- Martin, y Albert, 2010). En esta línea, Schimmarck (2005) encontró que independientemente de la intensidad, solo los estímulos emocionales de valencia negativa - independientemente de su intensidad- generaban una disminución del desempeño en tareas experimentales.

Una concepción actual acerca del procesamiento de los estímulos en contextos emocionales es la teoría de la doble vía (LeDoux, 2015), que plantea que el procesamiento emocional de los estímulos visuales puede llevarse a cabo por medio de dos vías anatómica y cognitivamente diferenciables que actúan de manera coordinada: una más automática y rápida, y otra más lenta y compleja (LeDoux y Phelps, 2008; LeDoux, 2015). En la vía rápida se procesan principalmente estímulos de valencia negativa, puesto que la necesidad de responder a ellos de manera rápida resulta adaptativamente más relevante que responder a estímulos de otra valencia (Carretié, Albert, López-Martín, y Tapia, 2009). En este circuito, la información resultante es pobre en términos de la calidad de la imagen conseguida, pero suficiente si se trata de detectar una potencial amenaza. Esta información es puesta al servicio de la amígdala para que emita una respuesta de manera rápida (Carretié, López-Martín, y Albert, 2010). Por lo tanto, esta primera vía sigue un recorrido directo del tálamo a la amígdala y se caracteriza por ser rápida, automática e incluso inflexible. Los resultados que apoyan esta línea se basan en estudios con técnicas de fMRI (Vuilleumier et al., 2001), que han encontrado una activación de la amígdala incluso cuando los estímulos emocionales aún no han sido atendidos. De esta manera, podría decirse que los estímulos emocionales, especialmente negativos, reciben prioridad de procesamiento (LeDoux, 2015), incrementando la demanda de recursos atencionales, a veces a expensas de la tarea primaria que el sujeto está llevando a cabo.

En la vía lenta el procesamiento también se inicia con la percepción de un estímulo y su ingreso al tálamo, solo que ahora recibe una mayor decodificación por diferentes áreas de asociación. Esta información más detallada, es enviada a la Corteza Pre-Frontal (CPF), que a su vez sigue el recorrido hacia la amígdala. Esta vía añade una latencia adicional al recorrido de la información desde el tálamo hasta la amígdala (Carretié et al., 2010), pero aporta beneficios adicionales dado que posibilita, a través del paso por la corteza, un procesamiento cortical más complejo, y una representación detallada del 
estímulo que permite el procesamiento consciente del mismo (LeDoux y Phelps, 2008). En resumen, los estímulos emocionales negativos y amenazantes son procesados de manera automática, mientras que el resto de los estímulos emocionales podrían atravesar un procesamiento más complejo. Incluso, diversos estudios han mostrado que las reacciones a los estímulos emocionales pueden ser moduladas por diferentes variables tales como: (1) las propiedades del estímulo, (2) las características individuales y (3) la demanda de la tarea (Lichtenstein-Vidne, Henik, y Safadi, 2012; Okon-Singer, Tzelgov, y Henik 2007).

\section{Figura 1}

Las diferencias en la interferencia de los estímulos emocionales pueden ser explicadas por las propiedades del estímulo, las características individuales y la demanda de la tarea. Adaptado de Okon-Singer, Lichtenstein-Vidne y Cohen, 2013.

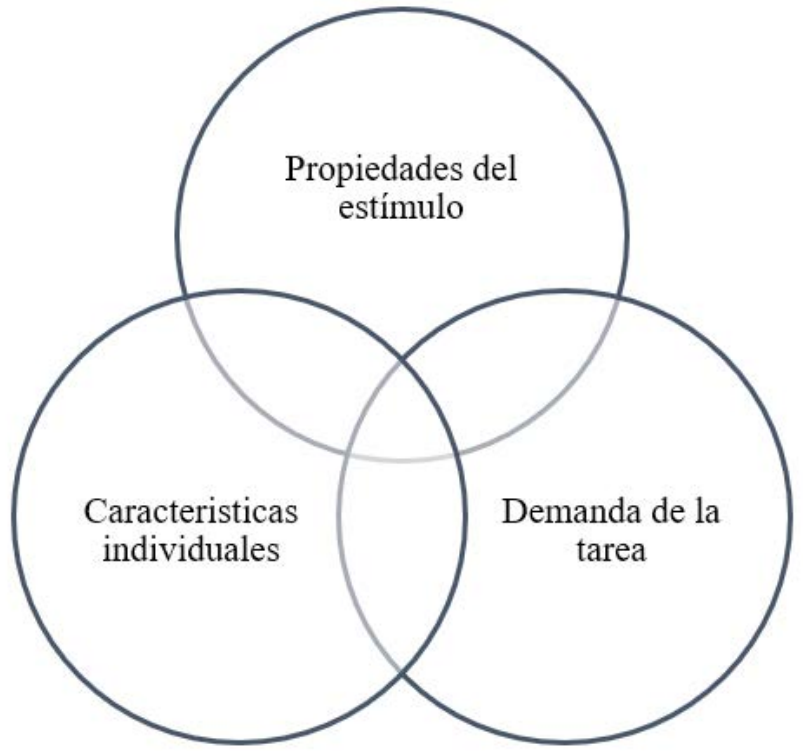

Por ejemplo, cuando una persona camina en su patio y de repente se encuentra con una araña, su reacción podría verse modulada por varios factores. En primer lugar, por el estímulo en sí mismo, ya que no es lo mismo encontrarse con una serpiente venenosa que con una pequeña araña de jardín. En segundo lugar, por la atención recibida y si el estímulo se encontraba en el foco atencional o no. De esta forma, la atención que recibirá el estímulo podría depender en gran medida si la persona estaba concentrada en una actividad exigente (por ejemplo, la lectura de un texto en otro idioma), descansando o si, por el contrario, estaba realizando una búsqueda activa de arañas en el patio. Por último, el procesamiento de este estimulo emocional y sus respuestas, también se verá afectado por las características individuales del receptor (por ejemplo, si la persona tiene una fobia específica a las arañas o no). A continuación, se revisará evidencia que podría sugerir que estos factores, podrían afectar, modular el procesamiento de los estímulos emocionales, y por lo tanto su respuesta.

Respecto de las propiedades de los estímulos, y tal como sucede con estímulos neutrales, los estímulos emocionales pueden clasificarse en estímulos verbales y no verbales. Los estímulos verbales, poseen la ventaja de permitir el control y manipulación de condiciones como el número de letras, frecuencia, idioma y familiaridad. Sin embargo, las palabras son controladas por el sistema léxico en primer lugar y luego desde allí acceden al sistema semántico. En cambio, los estímulos no verbales (estímulos visuales, auditivos, u olfativos) acceden directamente al sistema semántico, lo que los hace más adecuados para generar interferencia emocional debido a que estos estímulos acceden directamente a los esquemas emocionales más arraigados y automáticos (De Houwer y Hermans, 1994; Glaser y Glaser, 1989). A su vez, diferentes estudios han apoyado esta evidencia, demostrando que los estímulos visuales se procesan más rápido y presentan activaciones más fuertes que las palabras (Schacht y Sommer, 2009), e incluso que las respuestas emocionales ante palabras son menos intensas que ante estímulos visuales (Lees, Mogg, y Bradley, 2005). Por otro lado, el conjunto de estímulos visuales está conformado por imágenes dinámicas (clips de video) e imágenes estáticas (fotografías o dibujos). Los clips de video poseen validez ecológica, y provocan reacciones emocionales fuertes, aunque son difíciles de aplicar debido a los tiempos necesarios para evocar y medir diferentes aspectos 
del procesamiento emocional (Hasson, Malach, y Heeger, 2010). En el caso de las imágenes estáticas, se han utilizado conjuntos de expresiones faciales en forma de fotos o dibujos, y conjuntos de fotografías que representan aspectos de la vida en general (e.g., personas, paisajes, animales u objetos). Los estímulos emocionales como las caras se consideran biológica y socialmente importantes en comparación a otros estímulos emocionales (Price, 2010), y por lo tanto se las considera de un valor emocional alto. Por otro lado, las fotografías de contenido general (no específicas como las de expresiones faciales) poseen la capacidad de igualar las propiedades estimulares de los objetos reales o acontecimientos a que se refieren, dando lugar a la activación de representaciones cognitivas asociadas con respuestas emocionales fuertes (Bradley, Hamby, Löw, y Lang, 2007). En ese sentido se ha interpretado que si bien los dibujos (y también las palabras) son representaciones simbólicas de los objetos que componen el mundo que nos rodea, los estímulos visuales (como las imágenes), llegan a ser símbolos por su similitud física con los objetos reales, y por tanto se supone que los procesos por los cuales se reconoce, se comprende o se denomina un estímulo visual son similares a los procesos por los que se reconoce, comprende o denomina un objeto real (Glaser, 1992). Así, en la visualización de imágenes, todos los sujetos tienen una tarea de análisis común, de modo que la percepción de esos estímulos desencadena y determina respuestas emocionales de manera similar al patrón que tiene lugar ante los estímulos verdaderos (Lang, Greenwald, Bradley, y Hamm, 1993). En este sentido, este tipo de estímulos permite manipular y asegurarse de generar el efecto de interferencia, en las diferentes condiciones e incluso en diferentes sujetos. Asimismo, las imágenes o fotografías generales, tienen ventajas asociadas a su utilización experimental, porque posibilitan el control de su tamaño, resolución, brillo, frecuencia espacial y, sobre todo se destaca, su carácter no invasivo. Estas ventajas han motivado el desarrollo de diferentes bases estandarizadas de estímulos visuales cuyos contenidos representan un gran número de categorías, tales como escenas de la naturaleza (paisajes, plantas), objetos (utensilios, herramientas, comidas), caras humanas, animales, incluso hasta conjuntos de fotografías eróticas, entre otros.

En segundo lugar, las personas varían en la forma en que perciben y expresan sus emociones (Ekman y Davidson, 1994), y por lo tanto diversos autores sostienen que, al evaluar las reacciones emocionales, las diferencias y características individuales deben examinarse en lugar de considerarse ruido estadístico (Eugène et al., 2003). Los rasgos y características individuales pueden predisponer a las personas a procesar información que es congruente con esos rasgos (Compas et al., 2017). Por ejemplo, los participantes con fobias, muestran una disminución de desempeño frente a estímulos como fotografías de arañas y serpientes (Okon-Singer, Kofman, Tzelgov, y Henik, 2011). A su vez, pacientes con diagnóstico de trastorno de ansiedad y depresión a menudo dirigen su atención a contenidos negativos, significativamente en mayor medida que las personas sin este tipo de problemas (Mathews y MacLeod, 2005). Uno de los aportes destacados en esta línea es el meta-análisis de Bar-Haim, Lamy, Pergamin, Bakermans-Kranenburg y Van Ijzendoorn (2007), quienes concluyen que existen sesgos atencionales hacia información emocional de contenido amenazante en adultos con diferentes trastornos de ansiedad. Por este motivo considerar las características individuales de los participantes al realizar estudios experimentales, resulta una condición necesaria para realizar generalizaciones sobre el efecto de interferencia, debido a que estas características podrían afectar cómo se produce la interferencia.

Por último, respecto a los factores vinculados con las demandas de las tareas, se destacan por un lado la relevancia de los estímulos, y por otro lado la carga perceptual. Respecto de la relevancia para la tarea de los estímulos, la mayoría de las tareas experimentales emplean diferentes tipos de distractores para generar el efecto de interferencia: relevantes e irrelevantes. Los distractores 'relevantes' -generalmente denominados sólo distractores- son aquellos que comparten una o más características como forma y/o color con el target, y que, por su proximidad con el mismo (según el tipo de tarea), ingresan al foco atencional (Lavie, 1995). Sin embargo, también pueden distinguirse los estímulos enteramente irrelevantes. Según Forster y Lavie (2008), los distractores enteramente irrelevantes son aquellos que: (1) presentan una localización diferente al target y a los distractores relevantes, es decir por fuera del foco atencional, (2) no comparten ningún atributo visual con el target ni con los distractores relevantes, y (3) no se asocian a ningún conjunto de respuestas posibles. Forster y Lavie $(2008,2016)$ señalan que las personas en la vida cotidiana a menudo se distraen por estímulos que son totalmente irrelevantes para la tarea y que deben ignorarse. En este sentido, este tipo de estímulos -enteramente irrelevantes a la tarea- podrían considerarse los distractores ideales para generar el efecto de interferencia.

Además de la distinción entre distractores, Lavie (1995) destaca que también debe considerarse la carga perceptual en la realización de tareas cognitivas, la cual refiere a la complejidad y el número 
de unidades presentes en el display de estímulos (Lavie y Tsal, 1994). La manipulación de la carga perceptual en diferentes tareas mostró de manera consistente, que, independientemente de la separación entre el target y los distractores relevantes (1.4, 2.1 ó 2.9 grados), el efecto de interferencia de los distractores disminuía sustancialmente en función de la carga perceptual. Estos resultados mostraron que, a mayor carga perceptual, la susceptibilidad a la interferencia por información irrelevante no se producía. Por lo tanto, el procesamiento de los distractores irrelevantes no ocurre en tareas con alta carga perceptual (e.g., un número de distractores elevado, y/o un conjunto de características de los estímulos con bajo nivel de discriminación entre sí). De esta manera la baja carga perceptual de la tarea constituye una condición necesaria para que se genere el efecto de interferencia producido por distractores, ya sean estos emocionales o neutrales (Lavie, 2005). Si los estímulos emocionales no ingresan al sistema cognitivo, el efecto experimental de interferencia - en contextos emocionales y neutrales- no podría producirse.

\section{Conclusiones}

La manera en que los sujetos seleccionan y permanecen enfocados en la información relevante ante la presencia de información irrelevante resulta crucial para un comportamiento adaptativo. En un ambiente de estimulación continua y cambiante seleccionar un conjunto de estímulos (los relevantes) y descartar los que no son de nuestro interés (los irrelevantes) resulta fundamental para el logro de metas y objetivos. Por ejemplo, mantener la concentración en un ambiente de trabajo ruidoso, evitar la distracción generada por carteles publicitarios llamativos mientras conducimos, no poder dejar de pensar en la salida de fin de semana mientras estudiamos para un examen; todos estos comportamientos requieren la activación de procesos complejos, tales como la inhibición, cuya principal función consiste en atenuar el efecto de la interferencia (Canet Juric et al., 2016; Diamond, 2013). En este sentido, cuanto mayor es la interferencia, mayor es el esfuerzo que debe realizar la inhibición para contrarrestarla. En la actualidad, si bien existen distintas propuestas teóricas destinadas a explicar este efecto (ver MacLeod, Dodd, Sheard, Wilson, y Bibi, 2003); una de las propuestas más aceptadas es la que propone a la inhibición como principal mecanismo explicativo. En este sentido se entiende que el incremento en los TR registrado en condiciones de conflicto se debe a la intervención específica de la inhibición, proceso que se activa con el objeto de controlar o contrarrestar el efecto de la información irrelevante. Incluso, tal como explican Hofmann, Schmeichel y Baddeley (2012), el rol específico de la inhibición consiste en funcionar como un escudo protector contra la interferencia de pensamientos, comportamientos e incluso de las emociones.

Al respecto, las conceptualizaciones que consideran a la inhibición como un constructo multidimensional se han basado en la perspectiva formal y temporal de la interferencia para distinguir o clasificar los diferentes procesos inhibitorios. Por ejemplo, Diamond $(2013,2016)^{1}$, diferencia un proceso inhibitorio - la inhibición perceptual- que ocurre a nivel perceptivo y se encarga de suprimir o eliminar la interferencia coactiva o concurrente que generan los estímulos del ambiente externo sobre las tareas en curso. En este contexto, este proceso suprime la interferencia de estímulos irrelevantes de ambos dominios, neutrales o emocionales, que se encuentran actualmente presentes, o de manera concurrente, con el target u objetivo. Otro de los procesos inhibitorios distinguidos y asociado con la perspectiva temporal y formal de la interferencia es la inhibición cognitiva. La inhibición cognitiva, es el proceso encargado de contrarrestar la interferencia sobre representaciones irrelevantes (pensamientos, recuerdos no deseados) que ya han ingresado al sistema cognitivo. Pueden ser representaciones totalmente irrelevantes e intrusivas, o representaciones que en algún momento fueron relevantes, pero con el cambio de objetivos ya no lo son. Así, este proceso inhibitorio es el encargado del control o resistencia a la interferencia proactiva o retroactiva, es decir de información neutral o emocional previa que puede dificultar o entorpecer el procesamiento de información actual. Por último, se distingue la inhibición comportamental o de respuestas, encargada de suprimir una respuesta motora que compite con otra respuesta motora en relación a un comportamiento orientado a objetivos. Este proceso inhibitorio se encarga de contrarrestar la interferencia de otra respuesta prepotente neutral o emocional que se presenta en el mismo momento, pero, resulta incompatible con una respuesta que resulta más adecuada. A pesar de que el efecto de interferencia y el funcionamiento inhibitorio se han evaluado prioritariamente en contextos neutrales, son escasas las investigaciones que abordan su análisis en contextos emocionalmente salientes.

\footnotetext{
${ }^{1}$ Los nombres con los que se denominan a los distintos procesos inhibitorios pueden variar según los autores (Canet-Juric, Introzzi, y Zamora, 2016).
} 
Respecto a la interferencia en contextos emocionales, la investigación resulta contradictoria y difícil de interpretar. Si bien, la captura de recursos atencionales y de procesamiento por parte de la información emocionalmente saliente significa una ventaja cuando la emoción es relevante para la tarea, ya que impulsa un procesamiento más profundo y más efectivo de esos estímulos, cuando no es relevante para la tarea, este procesamiento automático y obligatorio convierte a las situaciones emocionalmente salientes en poderosas fuentes de interferencia que compiten con la información relevante, empeorando la ejecución de la tarea en curso. Si bien resulta esperable que en contextos emocionales se produzca un mayor efecto de interferencia en la tarea, muchos estudios no encuentran diferencias en función del contexto o dominio de aplicación o incluso observan que el desempeño en contextos emocionales mejora (e.g., Rebetez, Rochat, Billieux, Gay, y Van der Linden, 2015; Reveillon et al., 2018; Zamora, Introzzi, Vernucci, del Valle, y Richard's, 2020).

En este sentido, la consideración de factores tales como las propiedades del estímulo (e.g., color, iluminación, contraste y valencia), el modelo de emoción en el que se sustentan (dimensional o discreto), las características individuales de los participantes (si procesan de manera diferencial algún tipo de estímulo), e incluso las diferentes demandas de la tarea podrían resultar herramientas esclarecedoras para analizar el efecto de interferencia en ambos contextos ( es decir, con estímulos emocionales y con estímulos neutrales). Incluso, la consideración de estos podría aportar resultados más robustos en lo que respecta la utilización de la interferencia en contextos emocionales, que si bien es un campo reciente de consideración crece de manera considerable.

\section{Referencias}

1. Bar-Haim, Y., Lamy, D., Pergamin, L., Bakermans-Kranenburg, M. J., \& Van ljzendoorn, M. H. (2007). Threat-related attentional bias in anxious and nonanxious individuals: a meta-analytic study. Psychological Bulletin, 133, 1-24. https://doi.org/10.1037/0033-2909.133.1.1

2. Bradley, M. M., Hamby, S., Löw, A., \& Lang, P. J. (2007). Brain potentials in perception: picture complexity and emotional arousal. Psychophysiology, 44(3), 364-373. https://doi.org/10.1111/ j.1469-8986.2007.00520.x

3. Canet-Juric, L., Introzzi, I., \& Zamora, E. (2016). Inhibición o inhibiciones: No siempre inhibimos lo mismo. En I. Introzzi \& L. Canet Juric (Comps.), ¿Quién dirige la batuta? Funciones Ejecutivas: herramientas para la regulación de la mente, la emoción y la acción (pp. 40-59), Mar del Plata: EUDEM.

4. Carretié, L., Albert, J., López-Martín, S., \& Tapia, M. (2009). Negative brain: an integrative review on the neural processes activated by unpleasant stimuli. International Journal of Psychophysiology, 71, 57-63. https://doi.org/10.1016/j.ijpsycho.2008.07.006

5. Carretié, L., López-Martín, S., \& Albert, J. (2010). Papel de la corteza prefrontal ventromedial en la respuesta a eventos emocionalmente negativos. Revista de Neurología, 50, 245-252. https:// doi.org/10.33588/rn.5004.2009292

6. Casey, B. J. (2000). Disruption of inhibitory control in developmental disorders: A mechanistic model of implicated frontostriatal circuitry. En R. S. Siegler \& J. L. McClelland (Eds.), Mechanisms of cognitive development: The Carnegie Symposium on cognition (Vol. 28, pp. 327-349). Hillsdale, $\mathrm{NJ}$ : Erlbaum.

7. Cohen, A. O., Dellarco, D. V., Breiner, K., Helion, C., Heller, A. S., Rahdar, A., ... \& Casey, B. J. (2016). The impact of emotional states on cognitive control circuitry and function. Journal of Cognitive Neuroscience, 28, 446-459. https://doi.org/10.1162/jocn_a 00906

8. Cohen, N., \& Henik, A. (2012). Do irrelevant emotional stimuli impair or improve executive control?.Frontiers in Integrative Neuroscience, 6, 1-4. https://doi.org/10.3389/fnint.2012.00033

9. Compas, B. E., Jaser, S. S., Bettis, A. H., Watson, K. H., Gruhn, M. A., Dunbar, J. P., ... \& Thigpen, J. C. (2017). Coping, emotion regulation, and psychopathology in childhood and adolescence: A meta-analysis and narrative review. Psychological Bulletin, 143, 939-991. https://doi.org/10.1037/ bul0000110

10. Compton, R. J., Banich, M. T., Mohanty, A., Milham, M., Herrington, J., Miller, G., ...\& Heller, W. (2003). Paying attention to emotion: an fMRI investigation of cognitive and emotional Stroop tasks. Cognitive, Affective, \& Behavioral Neuroscience, 3, 81-96. https://doi.org/10.3758/CABN.3.2.81

11. Dempster, F. N. (1993). Resistance to interference: Developmental changes in a basic processing dimension. En M. L. Howe \& R. Pasnak (Eds.), Emerging themes in cognitive development (Vol. 1, pp. 3-27). New York: Springer-Verlag.

12. Diamond, A. (2013). Executive functions. Annual Review of Psychology, 64, 135-168. https://doi. org/10.1146/annurev-psych-113011-143750 
13. Diamond, A. (2016). Why improving and assessing executive functions early in life is critical. En J. A. Griffin, P. McCardle \& L. S. Freund (Eds.), Executive function in preschool-age children: Integrating measurement, neurodevelopment, and translational research (pp. 11-43). Washington, DC: American Psychological Association. https://doi.org/110.13140/RG.2.1.2644.6483

14. Edwards, W. H. (2010). Motor Learning and Control: From Theory to Practice. Belmont, California: Cengage Learning.

15. Ekman, P., \& Davidson, R. J. (Eds.). (1994). The nature of emotion. New York: Oxford University Press.

16. Eriksen, B. A., \& Eriksen, C. W. (1974). Effects of noise letters upon the identification of a target letter in a non-search task. Perception \& Psychophysics, 16, 143-149. https://doi.org/10.3758/ BF03203267

17. Eugène, F., Lévesque, J., Mensour, B., Leroux, J.-M., Beaudoin, G., Bourgouin, P., \& Beauregard, M. (2003). The impact of individual differences on the neural circuitry underlying emotion. Neurolmage, 19, 354-364. https://doi.org/10.1016/s1053-8119(03)00121-6

18. Forster, S., \& Lavie, N. (2008). Failures to ignore entirely irrelevant distractors: The role of load. Journal of Experimental Psychology: Applied, 14, 73-83. https://doi.org/10.1037/1076898X.14.1.73

19. Forster, S., \& Lavie, N. (2016). Establishing the attention-distractibility trait. Psychological Science, 27, 203-212 https://doi.org/10.1177/0956797615617761

20. Fox, E., Russo, R., Bowles, R., \& Dutton, K. (2001). Do threatening stimuli draw or hold visual attention in subclinical anxiety?. Journal of Experimental Psychology: General, 130, 681-700. https://doi.org/10.1037//0096-3445.130.4.681

21. Glaser, W. R. (1992). Picture naming. Cognition, 42, 61-105. https://doi.org/10.1016/00100277(92)90040-O

22. Glaser, W. R., \& Glaser, M. O. (1989). Context effects in stroop-like word and picture processing. Journal of Experimental Psychology: General, 118, 13. https://doi.org/10.1037/0096-3445.118.1.13

23. Gotlib, I. H., \& McCann, C. D. (1984). Construct accessibility and depression: An examination of cognitive and affective factors. Journal of Personality and Social Psychology, 47, 427-439. https:// doi.org/10.1037//0022-3514.47.2.427

24. Harnishfeger, K. K. (1995). The development of cognitive inhibition: Theories, definitions, and research evidence. En F. Dempster \& C. Brainerd (Eds.), Interference and inhibition in cognition (pp. 175-204). San Diego: Academic Press. https://doi.org/10.1016/B978-012208930-5/50007-6

25. Hasson, U., Malach, R., \& Heeger, D. J. (2010). Reliability of cortical activity during natural stimulation. Trends in Cognitive Sciences, 14, 40-48. https://doi.org/10.1016/j.tics.2009.10.011

26. Hofmann, W., Schmeichel, B. J., \& Baddeley, A. D. (2012). Executive functions and self-regulation. Trends in Cognitive Sciences, 16, 174-180. https://doi.org/10.1016/j.tics.2012.01.006

27. Houwer, J. D., \& Hermans, D. (1994). Differences in the affective processing of words and pictures. Cognition \& Emotion, 8, 1-20. https://doi.org/10.1080/02699939408408925

28. Iordan, A. D., Dolcos, S., \& Dolcos, F. (2013). Neural signatures of the response to emotional distraction: a review of evidence from brain imaging investigations. Frontiers in Human Neuroscience, 7, 200. https://doi.org/10.3389/fnhum.2013.00200

29. Jacoby, L. L., Debner, J. A., \& Hay, J. F. (2001). Proactive interference, accessibility bias, and process dissociations: Valid subject reports of memory. Journal of Experimental Psychology: Learning, Memory, and Cognition, 27, 686-700. https://doi.org/10.1037/0278-7393.27.3.686

30. Jones, A. D., Cho, R. Y., Nystrom, L. E., Cohen, J. D., \& Braver, T. S. (2002). A computational model of anterior cingulate function in speeded response tasks: Effects of frequency, sequence, and conflict. Cognitive, Affective, \& Behavioral Neuroscience, 2, 300-317. https://doi.org/10.3758/ CABN.2.4.300

31. Klein, G. S. (1964). Semantic power measured through the interference of words with color naming. American Journal of Psychology, 77, 576-588. https://doi.org/10.2307/1420768

32. Kron, A., Schul, Y., Cohen, A., \& Hassin, R. R. (2010). Feelings don't come easy: Studies on the effortful nature of feelings. Journal of Experimental Psychology: General, 139(3), 520-534. https:// doi.org/10.1037/a0020008

33. Lang, P. J., Greenwald, M. K., Bradley, M. M., \& Hamm, A. O. (1993). Looking at pictures: Affective, facial, visceral, and behavioral reactions. Psychophysiology, 30, 261-273. https://doi. org/10.1111/j.1469-8986.1993.tb03352.x 
34. Lavie, N. (1995). Perceptual load as a necessary condition for selective attention. Journal of Experimental Psychology: Human Perception and Performance, 21, 451-468. https://doi. org/10.1037/0096-1523.21.3.451

35. Lavie, N. (2005). Distracted and confused? Selective attention under load. Trends in Cognitive Sciences, 9, 75-82. https://doi.org/10.1016/j.tics.2004.12.004

36. Lavie, N., \& Tsal, Y. (1994). Perceptual load as a major determinant of the locus of selection in visual attention. Perception \& Psychophysics, 56, 183-197. https://doi.org/10.3758/BF03213897

37. Lavie, N., Hirst, A., De Fockert, J. W., \& Viding, E. (2004). Load theory of selective attention and cognitive control. Journal of Experimental Psychology: General, 133, 339-354. https://doi. org/10.1037/0096-3445.133.3.339

38. LeDoux, J. E. (2015). Anxious: Using the brain to understand and treat fear and anxiety. New York: Penguin.

39. LeDoux, J., \& Phelps, E. (2008). Emotional networks in the brain. En L. Feldman Barret, M. Lewis, \& J. Haviland-Jones (eds.) Handbook of Emotion, (3rd ed., pp. 159-179). New York: Guilford.

40. Lees, A., Mogg, K., \& Bradley, B. P. (2005). Health anxiety, anxiety sensitivity, and attentional biases for pictorial and linguistic health - threat cues. Cognition \& Emotion, 19, 453-462. https:// doi.org/10.1080/02699930441000184

41. Lichtenstein-Vidne, L., Henik, A., Safadi, Z., (2012). Task relevance modulates processing of distracting emotional stimuli. Cognition \& Emotion, 26, 42-52. https://doi.org/10.1080/02699931 .2011 .567055

42. MacLeod, C. M. (2007). The concept of inhibition in cognition. En D. S. Gorfein \& C. M. MacLeod (Eds.), Inhibition in cognition (pp. 3-23). Washington, DC, US: American Psychological Association. https://doi.org/10.1037/11587-001

43. MacLeod, C. M., Dodd, M. D., Sheard, E. D., Wilson, D. E., \& Bibi, U. (2003). In opposition to inhibition. En B. H. Ross (ed.), The psychology of learning and motivation - Advances in research and theory (Vol. 43, pp. 163-214). London: Academic Press. https://doi.org/10.1016/S00797421(03)01014-4

44. Mathews, A., \& MacLeod, C. (2005). Cognitive vulnerability to emotional disorders. Annual Review Clinical Psychology, 1, 167-195. https://doi.org/10.1146/annurev.clinpsy.1.102803.143916

45. Mauss, I. B., \& Robinson, M. D. (2009). Measures of emotion: A review. Cognition \& Emotion, 23, 209-237. https://doi.org/10.1080/02699930802204677

46. McGeoch, J. A., \& McDonald, W. T. (1931). Meaningful relation and retroactive inhibition. The American Journal of Psychology, 43, 579-588. https://doi.org/10.2307/1415159

47. McKenna, F. P., \& Sharma, D. (2004). Reversing the emotional Stroop effect reveals that it is not what it seems: the role of fast and slow components. Journal of Experimental Psychology: Learning, Memory, \& Cognition, 30, 382-392. https://doi.org/10.1037/0278-7393.30.2.382

48. McKenna, F. P., \& Sharma, D. (2004). Reversing the emotional Stroop effect reveals that it is not what it seems: the role of fast and slow components. Journal of Experimental Psychology: Learning, Memory, \& Cognition, 30, 382-392. https://doi.org/10.1037/0278-7393.30.2.382

49. Mishra, J., Anguera, J. A., Ziegler, D. A., \& Gazzaley, A. (2013). A cognitive framework for understanding and improving interference resolution in the brain. Progress in Brain Research, 207, 351-377. https://doi.org/10.1016/B978-0-444-63327-9.00013-8

50. Okon?Singer, H., Kofman, O., Tzelgov, J., \& Henik, A. (2011). Using international emotional picture sets in countries suffering from violence. Journal of traumatic stress, 24, 239-242. https://doi. org/10.1002/its.20600

51. Okon-Singer, H., Lichtenstein-Vidne, L., \& Cohen, N. (2013). Dynamic modulation of emotional processing. Biological Psychology, 92, 480-491. https://doi.org/10.1016/j.biopsycho.2012.05.010

52. Okon-Singer, H., Tzelgov, J., \& Henik, A., (2007). Distinguishing between automaticity and attention in the processing of emotionally significant stimuli. Emotion, 7, 147-157. https://doi. org/10.1037/1528-3542.7.1.147

53. Pratto, F., \& John, O. P. (1991). Automatic vigilance: the attention-grabbing power of negative social information. Journal of Personality and Social Psychology, 61, 380-391. https://doi. org/10.1037/0022-3514.61.3.380

54. Price, C. J. (2010). The anatomy of language: a review of $100 \mathrm{fMRI}$ studies published in 2009. Annals of the New York Academy of Science, 1191, 62-88. https://doi.org/10.1111/j.1749$\underline{6632.2010 .05444 . x}$ 
55. Rebetez, M. M. L., Rochat, L., Billieux, J., Gay, P., \& Van der Linden, M. (2015). Do emotional stimuli interfere with two distinct components of inhibition? Cognition \& Emotion, 29, 559-567. https://doi.org/10.1080/02699931.2014.922054

56. Réveillon, M., Lazeyras, F., Van Calster, L., Cojan, Y., Sander, D., Hüppi, P. S., \& Barisnikov, K. (2018, en prensa). Neural functional correlates of the impact of socio-emotional stimuli on performances on a flanker task in children aged 9-11 years. Neuropsychologia. https://doi.org/10.1016/j. neuropsychologia.2018.04.004

57. Schacht, A., Sommer, W., (2009). Emotions in word and face processing: early and late cortical responses. Brain and Cognition, 69, 538-550. https://doi.org/10.1016/j.bandc.2008.11.005

58. Schimmack, U. (2005). Attentional interference effects of emotional pictures: threat, negativity, or arousal? Emotion, 5, 55-66. https://doi.org/10.1037/1528-3542.5.1.55

59. Simon, J. R., \& Berbaum, K. (1990). Effect of conflicting cues on information processing: the 'Stroop effect'vs. the 'Simon effect'. Acta Psychologica, 73, 159-170. https://doi.org/10.1016/00016918(90)90077-S

60. Song, S., Zilverstand, A., Song, H., Uquillas, F. D. O., Wang, Y., Xie, C., ... \& Zou, Z. (2017). The influence of emotional interference on cognitive control: A meta-analysis of neuroimaging studies using the emotional Stroop task. Scientific Reports, 7, 1-9. https://doi.org/10.1038/s41598-017$\underline{02266-2}$

61. Underwood, B. J. (1957). Interference and forgetting. Psychological Review, 64, 49-60. https:// doi.org/10.1037/h0044616

62. van der Molen, M. W. (2000). Developmental changes in inhibitory processing: Evidence from psychophysiological measures. Biological Psychology, 54, 207-239. https://doi.org/10.1016/ S0301-0511(00)00057-0

63. Vuilleumier, P., Sagiv, N., Hazeltine, E., Poldrack, R. A., Swick, D., Rafal, R. D., \& Gabrieli, J. D. (2001). Neural fate of seen and unseen faces in visuospatial neglect: a combined event-related functional MRI and event-related potential study. Proceedings of the National Academy of Sciences, 98, 3495-3500. https://doi.org/10.1073/pnas.051436898

64. Williams, J. M. G., Mathews, A., \& MacLeod, C. (1996). The emotional Stroop task and psychopathology. Psychological Bulletin, 120, 3-24. https://doi.org/10.1037/0033-2909.120.1.3

65. Yiend, J. (2010). The effects of emotion on attention: A review of attentional processing of emotional information. Cognition \& Emotion, 24, 3-47. https://doi.org/10.1080/02699930903205698

66. Zamora, E. V, Introzzi, I., del Valle, M., Vernucci, S., Richard's, M. (2020). Perceptual inhibition of emotional interference. Applied Neuropsychology: Child, 9(3),215-229. https://doi.org/10.1080/2 1622965.2019 .1567340

67. Ziegler, D. A., Janowich, J. R., \& Gazzaley, A. (2018). Differential Impact of Interference on Internally-and Externally - Directed Attention. Scientific Reports, 8, 2498. https://doi.org/10.1038/ $\underline{\text { s41598-018-20498-8 }}$

RECIBIDO: 7 de octubre de 2019

MODIFICADO: 13 de diciembre de 2019

ACPTADO: 30 de junio de 2020 